\title{
Demonstration of Significance of Density Related to Developing Interest at Secondary Level Students in Chemistry (Case Study of Secondary level students)
}

\author{
*R. Azmat \\ *Department of Chemistry, University of Karachi \\ *Corresponding Author Email:rafiasaeed200@yahoo.com
}

\begin{abstract}
:
This report discusses the case study of secondary level students regarding their future interest in learning Chemistry as the main subject. Most students use particular words that it is not easy, can't follow, and can't understand. It was intended to develop an interest in Chemistry in young students. Therefore, a workshop was conducted where fundamental property Density was discussed concerning its application in the environment and pharmaceutics to show the vital role of introductory Chemistry in the presence of advanced technologies. It was demonstrated that density is a physical property that showed a relation between mass and volume. Its role in the current era of advancement is very significant, especially in the environment and pharmaceutics. It is used widely to indicate the purity of the substance leading to characterization and estimation of the composition of the mixture.
\end{abstract}

Keywords: Secondary level, students, Chemistry, Density, environment, and pharmaceutics

\section{INTRODUCTION:}

Investigators worldwide have observed extensive issues and practices in chemistry classrooms from several points of view. They also indicate escaping of students from secondary level related to teacher quality unable to satisfy them ${ }^{1}$. However, their investigation suggests several possible ways to develop an interest in learning chemistry at the secondary level. Though, research studies conducted at the secondary level, nationally and internationally in a chemistry classroom, have mainly concentrated on recognizing students' misapprehensions about an extensive series of chemistry concepts ${ }^{2}$. Typically, the studies interested in students' misapprehensions have used different diagnostic tools, such as test instruments, checklists, and so on, or conducting diagnostic interviews with students or teachers to elicit their fallacies and origins. Education of conceptual difficulties in chemistry more frequently observed that should not occur ${ }^{3}$. It should be for a specific purpose initially to motivate students to understand the subject quickly and creatively. Haitao ${ }^{4}$ reported about the principal reasons for the apparent deterioration in the experiment abilities of the student's majority in chemistry education are: 1) laboratory hours are not adequate, which is less than theory due to which students cannot concentrate on the experiment, 2) there is a gap in a week in practical classes and they are not close with each other, 3) lack of experimental reagents, glassware, and instruments in labs 4). The content, object, and necessities of chemistry teaching are not considered well and even not arranged for teacher training 5 . This case study aimed to develop students' interest by demonstrating a fundamental physical property of chemistry that is helpful in environmental studies to show Chemistry is a fundamental subject of Science.

\section{MATERIAL AND METHOD:}

$\mathrm{RD}$ bottle, weighing balance, a different form of water collected from a mineral water bottle, tap water while impure water prepared by adding some $\mathrm{NaCl}$.

\section{RESULTS AND DISCUSSION:}

Demonstration of the experiment for measuring density conducted in two ways intends to retain the students' interest in learning Chemistry. Initially, the RD bottle was taken and explained that this bottle is used to measure liquid density. It was explained that the density of water is taken as 1.00 , but this is a bit less and as $0.9998395 \mathrm{~g} / \mathrm{ml}$ at $4.0^{\circ}$ Celsius $\left(39.2^{\circ}\right.$ Fahrenheit). The specific gravity or relative density was also discussed. The other way to measure the density was also discussed that volume of liquid and mass of the liquid measure and record at weighing balance respectively to measure the density in the following way

1. Measure the weight of an empty RD bottle and fill the water in it remove any bubble from it, or alternatively

2. Measure the weight of water in grams.

3. Measure the volume of the water in $\mathrm{ml}$.

4. Calculate density $=\mathrm{M} / \mathrm{V}$ or Divide the mass by the volume.

5. You will then have the density of the water/liquid in $\mathrm{g} / \mathrm{ml}$.

6. Finally, it was discussed that if there is a change in the density of water/liquid from the standard value, it reflects the impurity in the water/ liquid 
After the demonstration and explanation of density, environmental and Pharmaceutical applications were discussed. It was discussed that density meters are also helpful in assessing the material porosity of a solid drug substance or determining the specific gravity of liquid samples. Estimating density in pharma helps to meet quality control standards and scrutinize the ultimate state of a final solid or suspended formulation while in the environmental helpful in estimating the purity of water/liquid.

\section{CONCLUSION:}

Although students showed some interest in this small session of two hours, they also point out difficulties in chemical equation balancing and other problems related to solving the numerical question in Chemistry. It was concluded that the students faced theoretical hurdles in learning subject-matter knowledge related to the Chemistry teacher. A detailed change in subject matter with teachers training in Chemistry is required to avoid the escaping tendency of the students from the Chemistry Classroom. It also illustrates the requirement of the multidimensional and energetic nature of the movement's indepth learning of scientific concepts of Chemistry at the secondary level.

\section{ACKNOWLEDGMENT:}

Author is very thankful to the group of the students for sparing their time to learn importance of Chemistry using example of Physical Property Density

\section{REFERENCES:}

1. Ali, T. (2012). SAGE Open, 2(2), 2158244012447299. Iqbal, H.

2. M., Nageen, T., Pell, A. W. (2008). Evaluation \& Research in Education, 21, 269-302.

3. Osborne, J., Simon, S., Collins, S. (2011). Journal of Research in Science Teaching, 25, 1049-1079.

4. LI Haitao https://en.cnki.com.cn/Article_en/CJFDTotal-YXSG200003022.htm. The Analysis of the Reasons for the Obvious Decline in the Experiment Abilities of the Students Majority in Chemistry Education

5. Tien Nguyen, (2018) Chemistry education moves from classroom to escape room. https://cen.acs.org/education/k\%E2\%80\%9312- 